\title{
Atuação fisioterapêutica em um indivíduo com lúpus eritematoso sistêmico associado à artrite reumatoide e à fibromialgia
}

\author{
Physiotherapy performance in an individual with systemic lupus \\ erythematosus associated with rheumatoid arthritis and fibromyalgia
}

Matheus Santos Gomes Jorge ${ }^{1}$, Karina Garbin¹, Poliana Luisa Müller ${ }^{1}$, Lia Mara Wibelinger ${ }^{1,2}$

${ }^{1}$ Universidade de Passo Fundo (UPF) - Passo Fundo (RS), Brasil.

${ }^{2}$ Geriatria e Gerontologia Biomédica, Pontifícia Universidade Católica do Rio Grande do Sul (PUCRS) - Porto Alegre (RS), Brasil.

DOI: http://dx.doi.org/10.7322/abcshs.v42i1.952

\begin{abstract}
RESUMO
Introdução: As doenças reumáticas podem manifestar-se de forma isolada ou concomitante, como, por exemplo, o lúpus eritematoso sistêmico, que pode associar-se à artrite reumatoide e à fibromialgia, comprometendo a funcionalidade e a qualidade de vida. O objetivo deste estudo foi verificar os efeitos da fisioterapia na dor, na força de preensão palmar, no equilíbrio postural, no nível de incapacidade e na qualidade de vida de um indivíduo com lúpus eritematoso sistêmico associado à artrite reumatoide e à fibromialgia. Relato de caso: Mulher, 56 anos, diagnosticada com lúpus eritematoso sistêmico, artrite reumatoide e fibromialgia há 14 anos. Sua queixa principal era dor generalizada e intensa em diversos pontos do corpo. Foram avaliados a dor, a força de preensão palmar, o equilíbrio postural, o nível de incapacidade e a qualidade de vida. O indivíduo realizou 30 sessões de fisioterapia (cinesioterapia e hidrocinesioterapia), duas vezes por semana, entre os meses de agosto e novembro de 2015, na Clínica de Fisioterapia da Universidade de Passo Fundo. Após a intervenção, houve diminuição da dor em 5 pontos e da incapacidade em 30\%; aumento da força de preensão palmar em 2,2 kgf (mão direita) e 1,2 kgf (mão esquerda); e do equilíbrio postural em 6 pontos. Como consequência, a qualidade de vida do indivíduo melhorou. Conclusão: A fisioterapia demonstrou-se eficaz na diminuição da dor e da incapacidade, no aumento da força de preensão palmar bilateral e do equilíbrio postural e na melhora da qualidade de vida em um indivíduo com lúpus eritematoso sistêmico associado à artrite reumatoide e à fibromialgia.
\end{abstract}

Palavras-chave: lúpus eritematoso sistêmico; artrite reumatoide; fibromialgia; exercício; fisioterapia; hidroterapia.

\begin{abstract}
Introduction: Rheumatic diseases may occur singularly or in concomitant way, such as systemic lupus erythematosus, that may be associated with rheumatoid arthritis and fibromyalgia, compromising the functionality and quality of life. The aim of this study was to investigate the effects of physiotherapy on pain, handgrip strength, postural balance, incapacity level and quality of life in an individual with systemic lupus erythematosus associated with rheumatoid arthritis and fibromyalgia. Case report: Woman, 56 years old, diagnosed with systemic lupus erythematosus, rheumatoid arthritis and fibromyalgia for 14 years. Her main complaint was widespread and intense pain in several points of the body. We evaluated pain, handgrip strength, postural balance, incapacity level and quality of life. The individual performed 30 sessions of physiotherapy (kinesiotherapy and hydrokinesiotherapy), twice a week, between August and November 2015, at Physiotherapy Clinic of the University of Passo Fundo. After the intervention, there was reduction of pain by 5 points and incapacity by $30 \%$, increased handgrip strength in 2,2 kgf (right hand) and 1,2 kgf (left hand) and postural balance in 6 points. As a consequence, the individual's quality of life improved. Conclusion: The physiotherapy proved to be effective in reducing pain and disability, increasing bilateral handgrip strength and postural balance and improving the quality of life of an individual with systemic lupus erythematosus associated with rheumatoid arthritis and fibromyalgia
\end{abstract}

Keywords: lupus erythematosus, systemic; arthritis, rheumatoid; fibromyalgia; exercise; physical therapy specialty; hydrotherapy.

Recebido em: 18/06/2016

Revisado em: 02/09/2016

Aprovado em: 21/09/2016

Autor para correspondência: Matheus Santos Gomes Jorge - Faculdade de Educação Física e Fisioterapia, Universidade de Passo Fundo - BR 285 - São José CEP: 99052-900 - Passo Fundo (RS), Brasil - E-mail: mathjorge5@gmail.com

Conflito de interesse: nada a declarar.

Fonte de financiamento: Fundação de Amparo à Pesquisa do Estado do Rio Grande do Sul (FAPERGS). 


\section{INTRODUÇÃO}

Algumas doenças reumáticas podem cursar em associação, como é o caso do lúpus eritematoso sistêmico (LES), que pode associar-se à artrite reumatoide (AR) e à fibromialgia (FM). De acordo com a literatura atual, o número de casos de associação entre as doenças supracitadas não chega a $15 \%$ dos relatos ${ }^{1}$.

O LES é uma patologia inflamatória, crônica, autoimune e de etiologia multifatorial, caracterizada por manifestações articulares, cutâneas, renais, vasculares, serosas e nervosas ${ }^{2}$, podendo provocar diminuição da mobilidade, da força muscular, do condicionamento físico e da qualidade do sono, bem como aumento da rigidez articular, da dor e da fadiga, resultando em um possível estado de depressão e impacto na qualidade de vida ${ }^{3}$. A AR e a FM são doenças idiopáticas e crônicas que provocam quadros dolorosos intensos e, por meio de agravos osteomioarticulares importantes, impactam a funcionalidade e a qualidade de vida de seus portadores ${ }^{4,5}$.

O tratamento das doenças reumáticas baseia-se no uso de medicamentos, como anti-inflamatórios não esteroidais, imunossupressores ou imunomoduladores, antidepressivos, entre outros, com o objetivo de minimizar as manifestações articulares e extra-articulares causadas pelas patologias em questão ${ }^{6}$. Adicionalmente, a fisioterapia também pode ser um método eficaz no tratamento de doenças reumáticas. Entre os benefícios, encontram-se a atenuação ou o controle dos sintomas causados pela doença, como, por exemplo, a diminuição da dor, a melhora da força muscular e a promoção de ganhos funcionais; consequentemente, ocorre a melhora da qualidade de vida dos indivíduos $\operatorname{acometidos}^{4,7}$.

Visto isso, este estudo objetivou verificar os efeitos de um plano de tratamento fisioterapêutico em um indivíduo com LES, AR e FM.

\section{RELATO DE CASO}

Trata-se de um estudo de caso intervencionista, que faz parte de um projeto guarda-chuva denominado "Efeitos do tratamento fisioterapêutico em pacientes com doenças reumáticas", aprovado pelo Comitê de Ética em Pesquisa em Seres Humanos da Universidade de Passo Fundo, sob protocolo no 348.381.

Participou do estudo um indivíduo do gênero feminino, 56 anos de idade, com diagnóstico médico de LES, AR e FM há 14 anos. Também apresentava comorbidades, como cardiopatia e hipotireoidismo, e tinha histórico de doença reumática na família. Sua queixa principal era dor generalizada. Fazia uso de sete diferentes medicamentos contínuos. Após a assinatura do Termo de Consentimento Livre e Esclarecido (TCLE), realizou 30 sessões de fisioterapia, duas vezes por semana (sendo a primeira por meio da cinesioterapia e a segunda por meio da hidrocinesioterapia), entre os meses de agosto e novembro de 2015, com duração de aproximadamente uma hora, na Clínica de Fisioterapia da Faculdade de Educação Física e Fisioterapia, da Universidade de Passo Fundo, Passo Fundo, RS.
A avaliação pré-intervenção fisioterapêutica envolveu a coleta de dados (como antropometria, história da doença atual, entre outros) e uma série de avaliações que consistiu na mensuração da dor por meio do mapa corporal de dor e da escala visual analógica de dor; da força de preensão palmar por meio da dinamometria manual; do equilíbrio postural por meio da escala de equilíbrio de Berg; da incapacidade por meio do Índice Oswestry 2.0 de Incapacidade; e da qualidade de vida por meio do Questionário de Qualidade de Vida Medical Outcomes Study 36 - Item Short-Form Health Survey. Depois disso, o indivíduo realizou um plano fisioterapêutico baseado em cinesioterapia e hidrocinesioterapia.

O plano cinesioterapêutico consistiu em: mobilização articular passiva dos punhos e dos ombros; exercício de "ponte" do método Bobath com bola suíça $(3 \times 10)$; rotação de tronco com bastão ( $3 \times 10)$; fortalecimento dos músculos flexores de ombro com bastão sem carga, progredindo até o uso de um halter de $1 \mathrm{~kg}$ ( $3 \times 10)$; fortalecimento dos músculos abdutores de ombro com halter de $1 \mathrm{~kg}$ (3x10); fortalecimento dos músculos extensores de ombro com faixa elástica rosa $(3 \times 10)$; fortalecimento dos músculos bíceps braquiais com halter de $500 \mathrm{~g}$ (3x10); fortalecimento das cinturas escapulares, obedecendo as diagonais de Kabat, com faixa elástica rosa, progredindo até a cor cinza (3x7); fortalecimento dos músculos flexores de quadril sem carga, progredindo até o uso de uma caneleira de $1 \mathrm{~kg}(3 \times 6)$; fortalecimento dos músculos abdutores e adutores de quadril com uma caneleira de $500 \mathrm{~g}$ ( $3 \times 10)$; exercício de equilíbrio em uma bola suíça; pompagens da região cervical; alongamentos finais dos principais grupos musculares dos membros superiores e inferiores e de tronco, de modo ativo assistido (15 segundos para cada grupo muscular).

O protocolo hidrocinesioterapêutico consistiu em: marcha estacionária (3 minutos); fortalecimento dos músculos flexores, extensores, adutores e abdutores de quadril, flexores e extensores de joelhos, flexores, extensores, adutores e abdutores de ombro com flutuadores (2x10); mini agachamentos, progredindo para mini agachamentos associados a fortalecimento dos músculos adutores de coxa com uma bola de voleibol entre as coxas (2x10); bicicleta estacionária em decúbito dorsal com flutuadores na região escapular (5 minutos); saltos alternados (2x10); exercício de sky cross-country (2 minutos); caminhada em torno da piscina de frente e de costas (2 voltas ao redor da piscina); alongamentos finais dos principais grupos musculares dos membros superiores e inferiores e de tronco, de modo ativo assistido (15 segundos para cada grupo muscular).

O indivíduo foi orientado a expirar durante a contração muscular a fim de obter maior recrutamento de fibras musculares, o que otimiza a performance do exercício. Adotou-se um intervalo de 30 segundos a 1 minuto entre as séries. Após as 30 sessões de intervenção fisioterapêutica, todos os parâmetros foram reavaliados.

A Tabela 1 apresenta os dados referentes às diversas variáveis avaliadas antes e após a intervenção. Em relação à dor, 
Tabela 1: Avaliação de dor, incapacidade, força de preensão palmar e equilíbrio pré e pós-intervenção fisioterapêutica

\begin{tabular}{|c|c|c|}
\hline & Pré-intervenção & Pós-intervenção \\
\hline Local da dor* & $\begin{array}{c}\text { - Região cervical; } \\
\text { - Região esternal; } \\
\text { - Região anterior do membro superior direito; } \\
\text { - Região anterior do antebraço esquerdo; } \\
\text { - Região lateral direita do tronco; } \\
\text { - Região posterior dos membros inferiores; } \\
\text { - Região lombar. }\end{array}$ & $\begin{array}{c}\text { - Região cervical; } \\
\text { - Região anterior dos membros superiores; } \\
\text { - Região lateral do quadril esquerdo; } \\
\text { - Joelhos; } \\
\text { - Região posterior da perna esquerda; } \\
\text { - Região plantar do pé esquerdo; } \\
\text { - Região lombar. }\end{array}$ \\
\hline Intensidade da dor ${ }^{\star *}$ & 10 & 5 \\
\hline $\begin{array}{l}\text { Pontuação do Índice de } \\
\text { Oswestry } 2.0 \text { de Incapacidade }\end{array}$ & $66 \%$ (aleijado) & $36 \%$ (incapacidade moderada) \\
\hline Força de preensão palmar da mão direita & $0,1 \mathrm{kgf}$ & $2,3 \mathrm{kgf}$ \\
\hline Força de preensão palmar da mão esquerda & $0,3 \mathrm{kgf}$ & $1,5 \mathrm{kgf}$ \\
\hline Pontuação do protocolo de equilíbrio de Berg & 35 & 41 \\
\hline
\end{tabular}

*segundo o mapa de dor corporal; **segundo escala visual analógica.

percebeu-se que, embora não fosse possível observar redução do número de pontos dolorosos, houve diminuição da intensidade geral da dor em 5 pontos, passando de um quadro de dor intensa para um quadro de dor moderada. Verificou-se, também, que a incapacidade do indivíduo apresentou considerável diminuição na fase pós-intervenção, com diferença de $30 \%$ em relação à fase pré-intervenção. Houve aumento da força de preensão palmar bilateral, com diferença de 2,2 kgf e 1,2 kgf entre as fases pré e pós-intervenção para as mãos direita e esquerda, respectivamente. E, finalmente, foi possível analisar que o indivíduo apresentou melhora do equilíbrio postural na fase pós-intervenção, com diferença de 6 pontos em relação à fase pré-intervenção.

A Tabela 2 apresenta os dados referentes à qualidade de vida do indivíduo estudado, de acordo com o Questionário de Qualidade de Vida Medical Outcomes Study 36 - Item Short-Form Health Survey. Observou-se melhora e/ou manutenção da qualidade de vida do indivíduo, sendo que o domínio "estado geral de saúde" apresentou o melhor resultado na pós-intervenção, seguido pelos domínios "capacidade funcional” e "limitação por aspectos físicos", respectivamente.

Tabela 2: Qualidade de vida pré e pós-intervenção fisioterapêutica, de acordo com o Questionário de Qualidade de vida SF-36

\begin{tabular}{|l|c|c|}
\hline Domínios & $\begin{array}{c}\text { Pré- } \\
\text { intervenção }\end{array}$ & $\begin{array}{c}\text { Pós- } \\
\text { intervenção }\end{array}$ \\
\hline Capacidade funcional & 0 & 25,0 \\
\hline Limitação por aspectos físicos & 0 & 25,0 \\
\hline Dor & 10,0 & 10,0 \\
\hline Estado geral de saúde & 20,0 & 65,0 \\
\hline Vitalidade & 80,0 & 75,0 \\
\hline Aspectos sociais & 37,5 & 37,5 \\
\hline Limitação por aspectos emocionais & 0 & 0 \\
\hline Saúde mental & 68,0 & 72,0 \\
\hline
\end{tabular}

\section{DISCUSSÃO}

A dor é uma das principais queixas encontradas em indivíduos com LES, AR ou $\mathrm{FM}^{3-5}$, e pode ser uma das principais causas de interferências em aspectos funcionais como a força de preensão palmar e o equilíbrio postural, causando incapacidade e impacto na qualidade de vida dos indivíduos. Neste estudo, a queixa principal foi dor generalizada e intensa (grau 10, de acordo com a escala visual analógica), que apresentava associação com as três doenças reumáticas. A dor foi identificada em diversos pontos, de acordo com o mapa de dor corporal. Dessa forma, pode-se considerar o combate à dor como uma estratégia para a restauração dos déficits de força de preensão palmar e de equilíbrio postural, visando diminuir a incapacidade e restaurar a qualidade de vida.

Os indivíduos com LES, AR e FM parecem beneficiar-se da fisioterapia que visa diminuir a dor e os processos inflamatórios; preservar a amplitude de movimento e atividade muscular; prevenir deformidades; restaurar o quadro cardiopulmonar e a funcionalidade; restabelecer o equilíbrio, a marcha e a propriocepção; além de proporcionar bem-estar e qualidade de vida ${ }^{3,4,8}$.

Um estudo com um indivíduo com LES e AR, do sexo feminino, que realizou 15 sessões de cinesioterapia convencional (fortalecimento, alongamento, equilíbrio, propriocepção), evidenciou a diminuição do quadro doloroso, a melhora da força muscular de membros inferiores e, consequentemente, a melhora da qualidade de vida ${ }^{9}$. Neste estudo, embora o número de pontos dolorosos permanecesse o mesmo na pós-intervenção, a intensidade geral da dor diminuiu consideravelmente, passando de intensa para moderada. Houve, também, aumento da força muscular (nesse caso, mensurada por meio da dinamometria manual) e melhora da qualidade de vida do indivíduo.

Acredita-se que o fato de o indivíduo ter FM, uma doença crônica e sistêmica, caracterizada por dor muscular generalizada, $\mathrm{e}$ apresentar pelo menos 11 tender points de 18 pré-definidos ${ }^{4}$ pode ter sido um fator que contribuiu para que o número de pontos dolorosos permanecesse o mesmo no período pós-intervenção. 
Aproximadamente 90\% dos portadores de LES apresentam artrite, com dor intensa e acometimento das mãos, punhos, joelhos e pés ${ }^{8}$. Concomitantemente a isso, alguns estudos evidenciam que a força de preensão palmar pode ser diminuída em indivíduos com doenças reumáticas se comparados com indivíduos sem a doença, como é o caso da FM e da $\mathrm{AR}^{9,10}$ - esta última apresenta correlação entre os valores da força de preensão palmar máxima e a atividade da doença ${ }^{10}$. Esses achados corroboram o presente estudo, visto que o indivíduo, que era portador das três doenças, relatou dor intensa (10, de acordo com a escala visual analógica) em diferentes pontos do mapa corporal e comprometimento da força de preensão palmar na fase pré-intervenção.

Um estudo de caso com um indivíduo com LES e AR envolveu atividades focadas em exercícios funcionais e de fortalecimento manual. Após as 15 sessões de intervenção fisioterapêutica, observou-se melhora da funcionalidade das mãos, aumento da amplitude de movimento de punhos e da força de preensão palmar bilateral e melhora da qualidade de vida do indivíduo ${ }^{11}$. Esses resultados corroboram o estudo atual, visto que nosso indivíduo, que possuía LES e AR associados (além de FM), apresentou melhora na força de preensão palmar e na qualidade de vida após 30 sessões de fisioterapia.

A associação de AR com outras doenças reumáticas, como LES, pode implicar em alterações na capacidade física, em virtude da presença de dor, da diminuição da força muscular, da propriocepção e da estabilidade mecânica, agravando o quadro de déficit do equilíbrio postural ${ }^{12}$. Ainda, outro estudo verificou que mulheres com FM, se comparadas a indivíduos sem a doença, apresentam déficit de equilíbrio postural e da autoconfiança por conta da dor e dos agravos causados pela patologia ${ }^{13}$. O mesmo foi observado no estudo atual, em que o indivíduo apresentava sério déficit do equilíbrio postural, que foi amenizado após a intervenção fisioterapêutica proposta.

Um indivíduo do sexo feminino, portador de LES, foi submetido a um plano que abordou diversas técnicas fisioterapêuticas, tais como cinesioterapia geral e respiratória, eletroterapia, hidrocinesioterapia, drenagem linfática e exercícios de coordenação, marcha e equilíbrio. Após 18 sessões de fisioterapia, constatou-se melhora no quadro doloroso, na amplitude de movimento e na qualidade de vida ${ }^{14}$. Apesar de nosso estudo ter abordado somente a cinesioterapia geral (que também incluiu exercícios de equilíbrio e coordenação) e respiratória em concomitância com a hidrocinesioterapia, também foram encontrados bons resultados com relação à diminuição da dor e à melhora da qualidade de vida do indivíduo estudado, que, além de LES, também apresentava AR e FM, doenças que causam dor e manifestações osteomioarticulares importantes.

Outro estudo hidrocinesioterapêutico com seis mulheres acometidas por FM demonstrou que, após 10 sessões de terapia aquática, a intervenção em grupo promoveu melhora no domínio "dor", abordado pelo Questionário de Qualidade de Vida Medical Outcomes Study 36 - Item Short-Form Health Survey, e no impacto causado pela doença na vida do indivíduo, de acordo com o Questionário de Impacto da Fibromialgia ${ }^{15}$. Conforme o presente estudo, o indivíduo demonstrou diminuição da intensidade da dor e melhora da qualidade de vida, além de outros benefícios, como a diminuição da incapacidade e o aumento da força de preensão palmar e do equilíbrio postural.

Acredita-se que uma possível limitação para o estudo tenha sido o pouco referencial encontrado na literatura a respeito de intervenções fisioterapêuticas aplicadas a indivíduos com doenças reumáticas associadas, o que restringiu a elaboração da metodologia desenvolvida neste estudo.

Nesse contexto, o plano de tratamento fisioterapêutico proposto para um indivíduo com LES associado à $\mathrm{AR}$ e à $\mathrm{FM}$ foi eficaz na redução da dor, no aumento da força de preensão palmar e na pontuação do equilíbrio postural, resultando, consequentemente, na redução do nível de incapacidade e na melhora ou manutenção dos domínios da qualidade de vida. Este estudo reforça os achados referentes aos benefícios da fisioterapia para o manejo das doenças reumáticas, sobretudo em indivíduos com doenças reumáticas associadas.

\section{REFERÊNCIAS}

1. Haliloglu S, Carlioglu A, Akdeniz D, Karaaslan Y, Kosar A. Fibromyalgia in patients with other rheumatic diseases: prevalence and relationship with disease activity. Rheumatol Int 2014;34(9):1275-80. http://dx.doi.org/10.1007/s00296-014-2972-8

2. Freire EAM, Souto LM, Ciconelli RM. Medidas de avaliação em lúpus eritematoso sistêmico. Rev Bras Reumatol. 2011;51(1):70-80.

3. Peres JMC, Tedde PFB, Lamari NM. Fadiga nos portadores de lúpus eritematoso sistemático sob intervenção fisioterapêutica. Mundo Saúde. 2006;30(1):141-5.
4. Batista JC, Borges AM, Wibelinger LM. Tratamento fisioterapêutico na síndrome da dor miofascial e fibromialgia. Rev Dor. 2012;13(2):170-4. http://dx.doi.org/10.1590/S1806-00132012000200014

5. Wibelinger LM, Molin VD, Myra RS, DeMarco M. Perfil de uma população portadora de artrite reumatoide em um município do interior do Rio Grande do Sul. Efdeportes. 2015;19(202).

6. Petean FC, Voltarelli JC, Donadi EA, Lousada Júnior P, Carvalho IF. Tratamento farmacológico das doenças reumáticas. Medicina. 1997;30(4):522-531. 
7. Myra RS, DeMarco M, Zanin C, Wibelinger LM. Kinesiotherapy for quality of life, pain and muscle strength of rheumatoid arthritis and systemic lupus erythematosus patient. Case report. Rev Dor. 2015;16(2):153-5. http://dx.doi.org/10.5935/1806-0013.20150029

8. Sociedade Brasileira de Reumatologia (SBR). Lúpus. 2011. Disponível em: http://www.reumatologia.com.br/PDFs/LES_Cartilha_ PDF_COMPLETO_2011.pdf. Acesso em: 21 jun. 2016.

9. Aparicio VA, Ortega FB, Heredia JM, Carbonell-Baeza A, Sjöström M Delgado-Fernandez M. Handgrip strength test as a complementary tool in the assessment of fibromyalgia severity in women. Arch Phys Med Rehabil. 2011;92(1):83-8.

http://dx.doi.org/10.1016/j.apmr.2010.09.010

10. Iop RR, Shiratori AP, Ferreira L, Borges Júnior NG, Domenech SC, Gevaerd MS. Capacidade de produção de força de preensão isométrica máxima em mulheres com artrite reumatoide: um estudo piloto. Fisioter Pesq. 2015:22(1):11-6. http://dx.doi.org/10.590/1809-2950/12371922012015

11. Myra RS, DeMarco M, Pancotte J, Rodrigues D, Secchin L, Sobral D, et al. Força de preensão palmar em um indivíduo portador de lúpus eritematoso sistêmico e artrite reumatóide: um estudo de caso. Efdeportes. 2015;20(209).

12. Bilberg A, Ahlmen M, Mannerkorpi K. Moderately intensive exercise in a temperate pool for patients with rheumatoid arthritis: a randomized controlled study. Rheumatology. 2005;44(4):502-8. http://dx.doi.org/10.1093/rheumatology/keh528

13. Santo ASE, Mango PC, Assumpção A, Sauer JF, Marques AP. Fibromyalgia: is there association between balance and pain? A pilot study. Fisioter Pesq. 2014;21(1):27-33. http://dx.doi.org/10.1590/1809-2950/341210114

14. Alves CT, Barros MFA, Oliveira EA, Carvalho AGC, Lucena NMG, Costa SML, et al. Abordagem fisioterapêutica ao portador de lúpus eritematoso sistêmico: relato de caso. Rev Bras Cienc Saúde. 2012;16(2):109-14. http://dx.doi.org/10.4034/RBCS.2012.16.s2.15

15. Jorge MSG, Myra RS, Schnornberger CM, Ranzi C, Wibelinger LM. Hidrocinesioterapia na dor e na qualidade de vida em indivíduos portadores de fibromialgia. Rev Inspirar. 2016;8(1):29-33. 